

Intrinsically switchable thin film bulk acoustic wave resonators

This document has been downloaded from Chalmers Publication Library (CPL). It is the author's version of a work that was accepted for publication in:

Applied Physics Letters (ISSN: 0003-6951)

Citation for the published paper:

Vorobiev, A. ; Gevorgian, S. (2014) "Intrinsically switchable thin film bulk acoustic wave resonators". Applied Physics Letters, vol. 104(22),

http://dx.doi.org/10.1063/1.4881141

Downloaded from: http://publications.lib.chalmers.se/publication/199412

Notice: Changes introduced as a result of publishing processes such as copy-editing and formatting may not be reflected in this document. For a definitive version of this work, please refer to the published source. Please note that access to the published version might require a subscription. 


\title{
Intrinsically switchable thin film bulk acoustic wave resonators
}

\author{
A. Vorobiev ${ }^{a)}$ and S. Gevorgian \\ Department of Microtechnology and Nanoscience, Chalmers University of Technology, SE-41296 Gothenburg, \\ Sweden
}

(Received 25 April 2014; accepted 21 May 2014; published online 3 June 2014)

\begin{abstract}
The concept of the frequency switching in the composite bulk acoustic wave (BAW) resonators based on the thin films of paraelectric phase ferroelectrics is demonstrated experimentally. The composite BAW resonators based on the $\mathrm{Ba}_{0.25} \mathrm{Sr}_{0.75} \mathrm{TiO}_{3} / \mathrm{SrRuO}_{3} / \mathrm{Ba}_{0.25} \mathrm{Sr}_{0.75} \mathrm{TiO}_{3}$ multilayer structure are fabricated and characterized. It is shown that the resonance frequency of the BAW resonators can be switched more than two times (from $3.6 \mathrm{GHz}$ to $7.7 \mathrm{GHz}$ ) by changing polarity of the $5 \mathrm{~V}$ dc bias voltage at one of the ferroelectric layers. The composite BAW resonators performance is analyzed using the theory of the dc field induced piezoelectric effect in the paraelectric phase ferroelectrics. C 2014 AIP Publishing LLC. [http://dx.doi.org/10.1063/1.4881141]
\end{abstract}

The electrically tunable thin film bulk acoustic wave (BAW) resonators, utilizing electric field induced piezoelectric effect in paraelectric phase ferroelectrics, have been intensively developed for the last five years. ${ }^{1-6}$ The BAW resonators with an improved $Q$-factor reveal a product of the $Q$ and frequency $Q f \approx 1900 \mathrm{GHz}$, which is already sufficient for practical applications in, e.g., tunable filters. ${ }^{6}$ The BAW resonators with record high tunability of the resonance frequency of $4.4 \%$ and effective electromechanical coupling coefficient of $10 \%$ have been reported recently. ${ }^{7}$ This tunability value is at the fundamental limit established in the theory of the field induced piezoelectric effect in the paraelectric phase ferroelectrics. ${ }^{8}$ However, for large scale industrial applications, for example, in the front ends of cellphones, even higher tunabilities are required.

Recently, an effective method to increase the BAW resonator tuning performance by using a composite structure consisting of two layers of paraelectric phase ferroelectrics has been proposed. ${ }^{9}$ Modelling demonstrates possibility of achieving about two times resonance frequency switching in a BAW resonator based, for example, on a $\mathrm{Pt} / \mathrm{SrTiO}_{3} / \mathrm{Pt} / \mathrm{SrTiO}_{3} / \mathrm{Pt}$ multilayer thin film structure. ${ }^{9}$ The resonance frequency switching is controlled by switching of mutual directions of the dc electric fields applied separately to the paraelectric layers. The field direction defines sign of the field induced piezoelectric coefficient. The unidirectional or oppositely directed fields result in excitation of acoustic eigenmodes with antisymmetric or symmetric mechanical displacement, respectively. Frequency of the lowest order antisymmetric eigenmode (mode1) is, approximately, two times lower than that of the symmetric eigenmode (mode2). The concept is similar to that used in an ultrasonic imaging transducer consisting of a stack of two plates of electrostrictive materials with polarizations controlled independently by the dc biases of selected polarity. ${ }^{10}$ The possibility of about two times resonance frequency switching using the lead magnesium niobate-lead titanate bulk ceramic stacks has been demonstrated experimentally. ${ }^{10}$ Recently, detailed analysis of the concept including a closed-form analytical

\footnotetext{
${ }^{\text {a) }}$ Author to whom correspondence should be addressed. Electronic mail: andrei.vorobiev@chalmers.se
}

formula for the complex impedance of the composite BAW resonators based on the paraelectric phase ferroelectric layers has been reported. ${ }^{11}$

In this work, we demonstrate experimentally the concept of the frequency switching in the composite BAW resonators based on the thin films of the paraelectric phase ferroelectrics. The resonator active area is composed of two paraelectric phase ferroelectric $\mathrm{Ba}_{0.25} \mathrm{Sr}_{0.75} \mathrm{TiO}_{3}$ (BST) films sandwiching a thin film oxide conductor $\mathrm{SrRuO}_{3}$ (SRO). The advantage of using the SRO as the intermediate electrode is that it possesses the cubic perovskite lattice structure, as the BST does, and reveals a very similar with the BST lattice parameter. ${ }^{12}$ This allows for in-situ quasi-epitaxial growth of the high quality multilayer stack of the BST/SRO/BST.

The composite BAW resonator test structures are fabricated as solidly mounted resonators on silicon substrates with resistivity of $20 \mathrm{k} \Omega \mathrm{cm}$. The Bragg reflector, consisting of $\lambda / 4 \mathrm{SiO}_{2} / \mathrm{W}$ layers $(\lambda=5.2 \mathrm{GHz})$ with thicknesses of $280 / 240 \mathrm{~nm}$, and $100 \mathrm{~nm}$ Pt bottom plate are deposited by magnetron sputtering. The $50 / 10 \mathrm{~nm}$ thick $\mathrm{TiO}_{2} / \mathrm{Ti}$ diffusion barrier/adhesion layers are deposited below the Pt bottom plate. The $\mathrm{Pt} / \mathrm{TiO}_{2} / \mathrm{Ti}$ bottom electrode stack is patterned to form openings below the dc contact pads, which allows for separate biasing of the paraelectric layers and eliminates parasitic resonances in the contact pad area. The patterning is done by means of $\mathrm{Ar}$ ion milling at $45^{\circ}$ tilt between the ion beam axis and the substrate surface to provide a sloped side wall of the opening edges. The BST/SRO/BST multilayer structure with thicknesses of 180/50/180 nm is grown in-situ in a pulsed laser deposition (PLD) system using stoichiometric targets. The targets are ablated by a $\mathrm{KrF}$ excimer laser beam with wavelength of $248 \mathrm{~nm}$, pulse duration of $30 \mathrm{~ns}$, and repetition rate of $10 \mathrm{~Hz}$, focused in a spot of $1 \times 4 \mathrm{~mm}^{2}$, which results in energy density of $1.5 \mathrm{~J} / \mathrm{cm}^{2}$. The BST and SRO films are grown in oxygen ambient at pressure of 0.4 millibars. The substrate temperature is maintained at $650^{\circ} \mathrm{C}$. The 20/90/10 nm thick Au/Al/Ti top electrode stack is deposited by e-beam evaporation. The top Au film is used for protection of the $\mathrm{Al}$ layer against etching by alkaline developers. A lift-off process is used to fabricate openings in the top electrode stack for separation of the piezo-active area 




FIG. 1. Schematic cross-sectional view of a composite resonator test structure. Applied sources of microwave and dc electric fields are also shown. Polarities of the dc biases shown correspond to the positive and negative signs of the piezoelectric coefficients induced in the top and bottom BST layers, respectively.

from the dc bias contact pad. Finally, the composite resonator test structure is formed, as shown in Fig. 1, by means of the photolithography and Ar ion milling of the top electrode followed by wet etching of the BST/SRO/BST structure down to the $\mathrm{Pt} / \mathrm{SiO}_{2}$ bottom plate. The BST films are selectively etched in a 1:1 buffered hydrofluoric acid in water solution. The SRO films are selectively etched in a $0.4 \mathrm{~mol} / \mathrm{l}$ solution of $\mathrm{NaIO}_{4}$ in water. ${ }^{13}$ A microphoto of the composite resonator test structure is shown in Fig. 2. Diameter of the piezo-active area is $40 \mu \mathrm{m}$.

The microwave input/output connection is achieved by using three-pin ground-signal-ground microprobes with direct contacting to the top electrode of the piezo-active area (signal) and the $\mathrm{Pt}$ bottom plate (ground) (see Figs. 1 and 2). Connection between the dc contact pad and the SRO layer is achieved through the top BST layer by means of the resistive/ capacitor divider (Fig. 1). The relatively large area of the dc contact pad provides effective dc connection to the SRO layer in the piezo-active area (Figs. 1 and 2). ${ }^{14}$ Two separate dc sources are used for applying the dc bias voltages to the top $\left(V_{\text {top }}\right)$ and bottom $\left(V_{\text {bot }}\right)$ BST layers (see Fig. 1). In experiments, the positive polarity of the $V_{\text {top }}$ is always applied to the top electrode of the piezo-active area, as shown in Fig. 1. It is

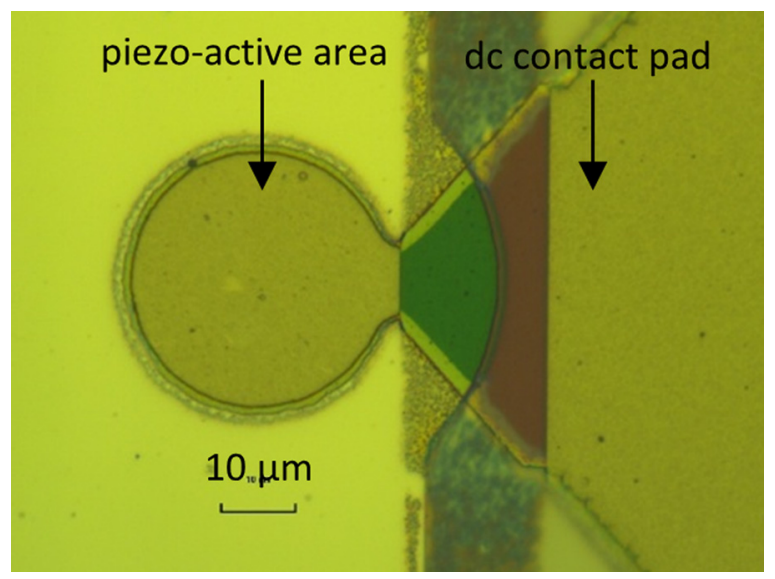

FIG. 2. Microphoto of the composite resonator test structure. assumed that this corresponds to the "down" direction of the dc electric fields $\left(E_{\mathrm{top}}\right)$ and positive sign of the induced piezoelectric coefficient in the top BST layer $\left(e_{\text {top }}>0\right)$. The polarity of the $V_{\text {bot }}$ is varied in the experiments. The positive polarity of the $V_{\text {bot }}$ applied to the dc contact pad corresponds to the "down" direction of the dc electric fields $\left(E_{\mathrm{bot}}\right)$ and positive sign of the induced piezoelectric coefficient in the bottom BST layer $\left(e_{\mathrm{bot}}>0\right)$. These polarities result in excitation of the resonance in the mode 1 . Alternatively, the negative polarity of the $V_{\text {bot }}$ applied to the dc contact pad corresponds to the "up" direction of the $E_{\mathrm{bot}}$ and negative sign of the induced piezoelectric coefficient in the bottom BST layer $\left(e_{\text {bot }}<0\right)$, as shown in Fig. 1. These polarities result in excitation of the resonance in the mode2. In experiments, the moduli of the $V_{\text {top }}$ and $V_{\text {bot }}$ are always kept equal to provide balanced conditions of the resonance modes.

The complex input impedance $Z=\operatorname{Re} Z+\mathrm{j} \operatorname{Im} Z$ and admittance of test structures are calculated via $S_{11}$ parameters measured using an Agilent N5230A vector network analyzer in the frequency range of $1-10 \mathrm{GHz}$. The modified Butterworth-Van Dyke (mBVD) circuit model is used for de-embedding the electrical loss associated mainly with the series resistance composed by the leading Pt bottom plate and the contact resistance between the Pt pad and the ground probe tips. The series resistance in the mBVD model is found, as the real part of impedance in the high frequency limit, to be $R_{\mathrm{S}}=1.65 \Omega$. ${ }^{14}$ This value of the $R_{\mathrm{S}}$ is consistent with resistance of a virtual ring section of the Pt bottom plate formed between the ground probe tips and the top electrode, which is calculated to be $1.05 \Omega$ assuming the bulk value of the Pt sheet resistance $1.06 \Omega{ }^{14}$ The microstructure of the BST films and the resonator multilayer stacks are analyzed using X-ray diffraction (XRD) technique. The XRD spectra are obtained using a Philips X'pert SW 3040 diffractometer equipped with a point $\mathrm{Cu} \mathrm{K}_{\alpha}$ radiation source, a thin film collimator, a rocking curve detector, and a Ni filter.

Fig. 3 shows combined XRD patterns of three partly completed resonator multilayer stacks including samples terminated by: (i) Pt bottom plate (labeled as substrate in Fig. 3), (ii) SRO layer, and (iii) top BST layer (see Fig. 1).

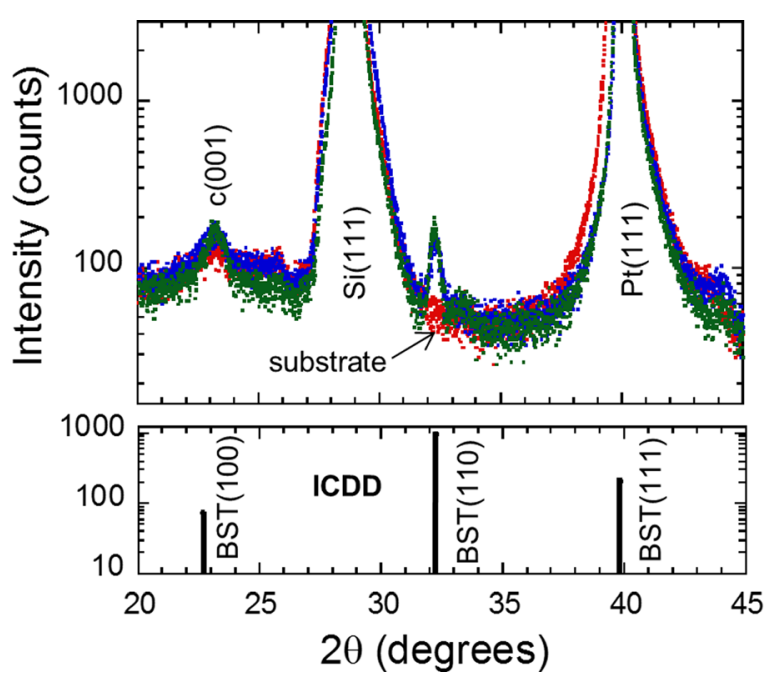

FIG. 3. XRD patterns of three partly completed resonator multilayer stacks including samples terminated by: (i) Pt bottom plate (labeled as substrate), (ii) SRO layer, and (iii) top BST layer. 




FIG. 4. Smith chart of the measured resonator $S_{11}$, without de-embedding $R_{\mathrm{s}}$, in the resonant mode $1\left(V_{\mathrm{bot}}=+5 \mathrm{~V}\right)$ and mode $2\left(V_{\mathrm{bot}}=-5 \mathrm{~V}\right)$.

Shown also are the standard XRD data of $\mathrm{Ba}_{0.256} \mathrm{Sr}_{0.744} \mathrm{TiO}_{3}$ powder (indexed according to the International Centre for Diffraction Data (ICDD) entry 01-089-8211). It can be seen that the resonator structures reveal no reflections from the BST(100) planes. The broad peak marked as c(001) is, most likely, related to the strained cristobalite. This indicates that the $\mathrm{SiO}_{2}$ Bragg reflector layers are, partially, in crystalline state. ${ }^{14}$ The BST(111) reflections can be masked by the strong $\operatorname{Pt}(111)$ peaks. The only reflections from the BST(110) orientation is observed. Similarity of the BST(110) peaks of the samples terminated by the SRO and the top BST layers indicate that the top BST layer has crystallinity similar to that of the bottom one. Therefore, one can assume symmetry of the composite structure properties and expect fair resonance balancing in the mode 2 .

Fig. 4 shows the Smith chart of the measured $S_{11}$, without de-embedding $R_{\mathrm{s}}$, in the resonant mode $1\left(V_{\mathrm{bot}}=+5 \mathrm{~V}\right)$ and mode $2\left(V_{\mathrm{bot}}=-5 \mathrm{~V}\right)$. Fig. 5 shows the impedance magnitude of the resonator versus frequency in both resonant modes, mode $1\left(V_{\mathrm{bot}}=+5 \mathrm{~V}\right)$ and mode $2\left(V_{\mathrm{bot}}=-5 \mathrm{~V}\right)$. It can be seen that in the mode 1 , at $V_{\mathrm{bot}}=+5 \mathrm{~V}$, the resonance is exited at frequency of, approximately, $3.6 \mathrm{GHz}$. No any other resonances are observed in the frequency range of $1-10 \mathrm{GHz}$. This resonance condition is defined by the same, positive, signs of the piezoelectric coefficients in each paraelectric layer $\left(e_{\mathrm{top}}>0\right.$ and $e_{\text {bot }}>0$ ) (Fig. 1). Both layers are simultaneously expanded or compressed under a microwave signal with anti-symmetric mechanical displacement. ${ }^{9,11}$ Therefore, the resonance frequency of the mode 1 is defined by the total thickness of the



FIG. 5. Impedance magnitude of the composite BAW resonator in the resonant mode $1\left(V_{\mathrm{bot}}=+5 \mathrm{~V}\right)$ and mode $2\left(V_{\mathrm{bot}}=-5 \mathrm{~V}\right)$. Shown also is simulated reflection coefficient of the Bragg reflector. resonator layered structure. In the alternative case, in the mode 2 , at $V_{\text {bot }}=-5 \mathrm{~V}$, the resonance is exited at frequency of, approximately, $7.7 \mathrm{GHz}$. This resonance condition is defined by the opposite signs of the piezoelectric coefficients in the paraelectric layers $\left(e_{\text {top }}>0\right.$ and $\left.e_{\text {bot }}<0\right)$ (Fig. 1). The difference in signs of the piezoelectric coefficients of the paraelectric layers, due to oppositely directed polarizations, results in $180^{\circ}$ phase shift between the piezoresponses of layers to the microwave signal. One of the layers is expanded another is compressed. Therefore, the existence of piezoelectric resonances of the antisymmetric displacement modes is prohibited and only symmetric modes (in particular, mode2) can be excited. ${ }^{9,11}$ The resonance frequency of the mode 2 is more than two times large than that of the mode1. Thus, the switching of the applied dc bias polarity $V_{\text {bot }}$ results in a change of excitation conditions for piezoelectric modes, which, in turn, allows for more than two times frequency switching (from $3.6 \mathrm{GHz}$ in the mode 1 to $7.7 \mathrm{GHz}$ in the mode 2 ). It can be seen from Fig. 5 that in the case of the mode 1 there is no any sign of the resonance at $7.7 \mathrm{GHz}$ since the symmetric displacement modes are prohibited. Alternatively, in the mode2, there is no resonance at $3.6 \mathrm{GHz}$, since the antisymmetric displacement modes are prohibited. Fig. 5 shows also the reflection coefficient of the Bragg reflector calculated assuming acoustic waves incident from an infinitely thick ferroelectric layer. As it can be seen, the resonance frequencies of both modes are within reflection band of the Bragg reflector.

Apart from the resonance frequency switching, the composite resonators possess analog tuning capability, which is an inherent property of any intrinsically tunable ferroelectric BAW resonators. Fig. 6 shows series and parallel resonance frequencies versus modulus of the bias voltage in both resonant modes and in the whole range of the applied voltages. The series $\left(f_{\mathrm{s}}\right)$ and parallel $\left(f_{\mathrm{p}}\right)$ resonance frequencies are defined at the maximum of the real part of the admittance and impedance, respectively. The tunability of the resonance frequency is defined as

$$
n_{\mathrm{s}, \mathrm{p}}=\frac{f_{0}-f_{\mathrm{s}, \mathrm{p}}}{f_{0}},
$$

where $f_{0}$ is the resonance frequency extrapolated to zero dc electric field. The tunabilities of the series and parallel resonance frequencies calculated using (1) are, approximately,



FIG. 6. Series $\left(f_{\mathrm{s} 1,2}\right)$ and parallel $\left(f_{\mathrm{p} 1,2}\right)$ resonance frequencies of the composite BAW resonator vs. modulus of the bias voltage in the resonant mode 1 and mode 2 . 
$1.0 \%$ and $0.5 \%$, respectively, for both mode 1 and mode 2 at $5 \mathrm{~V}$ of the dc bias voltage modulus. The lower tunability of the parallel resonance frequency is in agreement with the theory of the dc field induced piezoelectric effect. ${ }^{1}$ Tunabilities of the series resonance frequencies can be increased up to the theoretical limit of $3 \%-4 \%$ by selecting ferroelectric materials with higher linear electrostriction coefficient, $\mathrm{BiFeO}_{3}-\mathrm{BaTiO}_{3}$ solid solution, for example. ${ }^{7}$ The combination of the frequency switching and the analog tuning capability provides extended functionalities of the tunable two-layer composite BAW resonators, which may be useful, for example, for frequency band and channel selection in cellphones.

The quality factors of the composite resonators at series and parallel resonances are defined as ${ }^{14,15}$

$$
Q_{\mathrm{s}, \mathrm{p}}=\left.\frac{1}{2} f_{\mathrm{s}, \mathrm{p}} \frac{\partial \varphi_{\mathrm{de}}}{\partial f}\right|_{f=f_{\mathrm{s}, \mathrm{p}}},
$$

where $\varphi_{\mathrm{de}}=\operatorname{arctg}\left[\operatorname{Im} Z /\left(\operatorname{Re} Z-R_{\mathrm{S}}\right)\right]$ is the de-embedded phase angle. Fig. 7 shows quality factors versus modulus of bias voltage in the mode $1\left(Q_{\mathrm{s}, \mathrm{p} 1}\right)$ and mode $2\left(Q_{\mathrm{s}, \mathrm{p} 2}\right)$ calculated using (2). The $Q$-factors approach zero at low bias voltages due to the nature of the field induced piezoelectric effect. The $Q_{\text {s1 }}$ values at higher voltages are significantly larger than those of the $Q_{\mathrm{p} 1}$. This is because the definition (2) makes the $Q_{\text {s }}$ equal to the purely mechanical $Q$-factor. ${ }^{16}$ The $Q_{\mathrm{p} 1}$ values are reduced by additional contribution of the dielectric loss. ${ }^{16}$ It can be seen from Fig. 7 that in the mode 2 the $Q_{\mathrm{s} 2}$ and $Q_{\mathrm{p} 2}$ values are practically identical and, approximately, two times less than those in the mode1. This can be explained by the inverse dependence of the mechanical $Q$-factor on frequency. ${ }^{17,18}$ At higher frequencies, in the mode 2 , the mechanical loss dominates over the dielectric loss, which makes the $Q_{\mathrm{s} 2}$ and $Q_{\mathrm{p} 2}$ values comparable. As it can be seen, the $Q_{\mathrm{s} 1}$ values at high voltages approach maximum of, approximately, 100. This is a typical value for the conventional, non-composite, BAW resonators based on the BST films grown by PLD, operating at similar frequencies. ${ }^{19}$ It was shown that the $Q$-factor of the BAW resonators based on the ferroelectric films is, currently, limited by the mechanical loss associated with the wave scattering at reflection from relatively rough top interface. ${ }^{20}$ The BAW resonators based on the BST films with smoother surface, grown by magnetron sputtering, reveal $Q$-factors as high as 360 at $5.2 \mathrm{GHz}{ }^{6}$ Thus,



FIG. 7. Quality factors of the series $\left(Q_{\mathrm{s} 1,2}\right)$ and parallel $\left(Q_{\mathrm{p} 1,2}\right)$ resonances of the composite BAW resonator vs. modulus of bias voltage in the resonant mode 1 and mode 2 . the $Q$-factors of the composite resonators can be significantly improved via reduction of the ferroelectric film surface roughness, by using the magnetron sputtering, for example.

To summarize, we have demonstrated experimentally the concept of the frequency switching in the composite BAW resonators using thin films of paraelectric phase ferroelectrics. The fabricated composite $\mathrm{BAW}$ resonator based on the $\mathrm{Ba}_{0.25} \mathrm{Sr}_{0.75} \mathrm{TiO}_{3} / \mathrm{SrRuO}_{3} / \mathrm{Ba}_{0.25} \mathrm{Sr}_{0.75} \mathrm{TiO}_{3}$ multilayer structure reveals switching of the resonance frequencies more than two times, from $3.6 \mathrm{GHz}$ (mode 1 ) to $7.7 \mathrm{GHz}$ (mode2), by changing polarity of the $5 \mathrm{~V}$ dc bias voltage at one of the ferroelectric layers. The resonance frequency switching is combined with the analog tuning, which is found to be $1.0 \%$ and $0.5 \%$, for series and parallel resonance frequencies, respectively, in both mode 1 and mode 2 at the $5 \mathrm{~V}$ dc bias voltage modulus. The tunability can be increased up to the theoretical limit of $3 \%-4 \%$ by selecting ferroelectric material with higher linear electrostriction coefficient. The mechanical $Q$-factors of the composite resonators are up to 100 and 40 in the mode 1 and mode 2 , respectively. The $Q$-factors can be significantly increased by using the BST films with smoother surface, grown by magnetron sputtering, for example. The measured parameters make the composite BAW resonators very attractive, in particular, for application in advanced and emerging microwave communication systems.

This work was supported by Swedish Research Counsel Vetenskaprådet via projects VR-FBAR and CompFBAR.

${ }^{1}$ A. Noeth, T. Yamada, P. Muralt, A. K. Tagantsev, and N. Setter, IEEE Trans. Ultrason. Ferroelectr. Freq. Control 57, 379 (2010).

${ }^{2}$ S. A. Sis, V. Lee, J. D. Phillips, and A. Mortazawi, in IEEE MTT-S International Microwave Symposium Digest, 2012, p. 1.

${ }^{3}$ G. N. Saddik, J. Son, S. Stemmer, and R. A. York, J. Appl. Phys. 109, 091606 (2011).

${ }^{4}$ A. Volatier, E. Defay, M. Aid, A. N'hari, P. Ancey, and B. Dubus, Appl. Phys. Lett. 92, 032906 (2008).

${ }^{5}$ B. Ivira, A. Reinhardt, E. Defay, and M. Aid, in IEEE International Frequency Control Symposium, 2008, p. 254.

${ }^{6}$ A. Vorobiev and S. Gevorgian, Appl. Phys. Lett. 96, 212904 (2010).

${ }^{7}$ A. Vorobiev, S. Gevorgian, N. Martirosyan, M. Löffler, and E. Olsson, Appl. Phys. Lett. 101, 232903 (2012).

${ }^{8}$ A. Noeth, T. Yamada, A. K. Tagantsev, and N. Setter, J. Appl. Phys. 104, 094102 (2008).

${ }^{9}$ A. B. Kozyrev, A. K. Mikhaylov, S. V. Ptashnik, S. V. Zinoviev, P. K. Petrov, N. M. Alford, and T. Wang, Electron. Lett. 47(24), 1326 (2011).

${ }^{10}$ T. R. Gururaja, A. Shurland, and J. Chen, in IEEE Ultrasonics Symposium, 1997, p. 1659.

${ }^{11}$ S. Gevorgian and A. Vorobiev, IEEE Trans. Ultrason. Ferroelectr. Freq. Control 60, 795 (2013).

${ }^{12}$ P. Rundqvist, A. Vorobiev, and S. Gevorgian, J. Appl. Phys. 93, 1291 (2003).

${ }^{13}$ D. Weber, R. Vöfely, Y. Chen, Y. Mourzina, and U. Poppe, Thin Solid Films 533, 43 (2013).

${ }^{14}$ A. Vorobiev, S. Gevorgian, M. Löffler, and E. Olsson, J. Appl. Phys. 110, 054102 (2011).

${ }^{15}$ J. Kaitila, RF Bulk Acoustic Wave Filters for Communications, edited by K.-Y. Hashimoto (Artech House, Norwood, MA, 2009), p. 58.

${ }^{16} \mathrm{~A}$. Vorobiev and S. Gevorgian, Int. J. Microwave Wireless Technol. 5, 361 (2013).

${ }^{17}$ J. F. Rosenbaum, Bulk Acoustic Wave Theory and Devices (Artech House, Norwood, MA, 1988), p. 14.

${ }^{18}$ S. Gevorgian, A. Tagantsev, and A. Vorobiev, Tuneable Film Bulk Acoustic Wave Resonators (Springer, 2013), p. 46.

${ }^{19} \mathrm{~J}$. Berge and S. Gevorgian, IEEE Trans. Ultrason. Ferroelectr. Freq. Control 58, 2768 (2011).

${ }^{20}$ A. Vorobiev, J. Berge, S. Gevorgian, M. Löffler, and E. Olsson, J. Appl. Phys. 110, 024116 (2011). 